\title{
MONITORAMENTO DE PAVIMENTOS COM DISPOSITIVOS MÓVEIS
}

\author{
D. R. Bisconsini ${ }^{1}$; J. L. Fernandes Júnior ${ }^{2}$ \\ ${ }^{1,2}$ Escola de Engenharia de São Carlos, Universidade de São Paulo (EESC-USP), Departamento de Engenharia \\ de Transportes, Avenida Trabalhador são-carlense, 400 \\ danilorbisconsini@usp.bri , leomar@sc.usp.br²
}

Resumo: Diversas agências de transportes e universidades estão em busca de novas tecnologias para aprimorar a prática de levantamento de defeitos para a alimentação de Sistemas de Gerência de Pavimentos (SGP). Este estudo inclui a revisão, análise crítica e síntese de diversos estudos e desenvolvimentos que buscaram, a partir de diferentes métodos, avaliar a condição de pavimentos por meio de dados fornecidos por dispositivos móveis. O objetivo é apontar os principais métodos utilizados, vantagens e limitações dessas abordagens. Por meio do estudo realizado, conclui-se que os dispositivos móveis, com alguns cuidados e restriçốes, podem ser utilizados como ferramentas para a avaliação da condição de pavimentos em nível de rede, com potencial de ganho em confiabilidade dentro de alguns anos, em função do desenvolvimento de seus sensores e de seu uso em larga escala.

Palavras-chave: Gerência de pavimentos, Smartphones, Monitoramento de pavimentos.

\begin{abstract}
Several transportation agencies and universities are looking for new technologies for collecting data for Pavement Management Systems (PMS). This study includes the review, critical analysis and synthesis of several researches and developments that have sought, from different methods, to evaluate the condition of pavements through data provided by mobile devices. The objective is to indicate the main methods used with the advantages and limitations of these approaches. Through the study, it was concluded that mobile devices, with some cautions and restrictions, can be used as an assessment tool of pavement conditions at network-level, with the potential to gain reliability within some years, due to the development of its sensors and its large-scale use.
\end{abstract}

Keywords: Pavement management, Smartphones, Pavement monitoring. 


\section{Introduçáo}

O desenvolvimento de novas tecnologias é fundamental para o auxílio na tomada de decisóes administrativas, como o planejamento, programação e orçamento das atividades que envolvem um Sistema de Gerência de Pavimentos (SGP), o que inclui: 1) a identificação de projetos candidatos às intervençôes; priorização de projetos conforme medidas de desempenho, tráfego, custo dos usuários, entre outros parâmetros locais; 2) indicação de carências das agências de transportes de curto, médio e longo prazo; 3) auxílio em análises de estratégias de intervenção, com a verificação da condição atual e previsão futura da rede, de acordo com os recursos aplicados em cada caso [1].

Informações sobre a condição de pavimentos, coletadas periodicamente, são importantes no controle da evolução dos defeitos e um indicativo sobre as possíveis causas de deterioração de determinadas seçôes de pavimentos (fatores de tráfego, climáticos, construtivos, etc.). A partir desses dados, busca-se a melhor estratégia sobre os serviços de manutenção e reabilitação de pavimentos, contribuindo para uma gestão mais eficiente dos recursos públicos.

No levantamento de defeitos em pavimentos, é comum a utilização de sistemas sofisticados para a mensuração simultânea da irregularidade longitudinal e transversal de pavimentos, como os perfilômetros inerciais a laser e os sistemas do tipo scanner, estes últimos também utilizados para a avaliação da condição de outros defeitos. Entretanto, ambos os métodos demandam um alto custo de aquisição e operação.

Também são utilizados equipamentos estáticos de alta exatidão, mas pouco práticos para levantamentos em nível de rede. Outros procedimentos como os Levantamentos Visuais Contínuos (LVC) e levantamentos de defeitos por caminhamento são populares, principalmente, em países em desenvolvimento. No entanto, o primeiro tem como desvantagem a subjetividade inerente ao método e o segundo demanda tempo e uma equipe fixa e treinada para o serviço.

De modo geral, os métodos existentes apresentam um desequilíbrio entre custos, praticidade e exatidão, o que dificulta o levantamento contínuo de dados, importante para a alimentação de Sistemas de Gerência de Pavimentos (SGP). Por esse motivo, compreende-se o esforço de diversos estudos na busca de novas tecnologias para a melhoria das técnicas de monitoramento de pavimentos.

Órgãos públicos e universidades têm pesquisado a viabilidade do uso de sinais fornecidos por dispositivos móveis (tablets e smartphones) para a avaliação da condição de pavimentos, principalmente devido ao potencial que apresentam: baixo custo, praticidade e alta produtividade. $\mathrm{O}$ sistema poderia contribuir com a avaliação da condiçâo de rodovias rurais, mais especificamente da irregularidade longitudinal de pavimentos, e avaliação da condição de pavimentos urbanos, principalmente de buracos. 


\section{Metodologia}

Este estudo parte da revisão e análise crítica, de maneira estruturada e sintética, de diversas pesquisas e desenvolvimentos que buscaram, com base em diferentes métodos, avaliar a condição de pavimentos com dados fornecidos por dispositivos móveis.

\section{Resultados}

Para o levantamento de defeitos em pavimentos, dispositivos móveis são fixados no corpo de veículos (Fig. 1-a), de modo que um dos eixos do aparelho (Fig. 1-b), esteja posicionado o mais perpendicular possível do eixo longitudinal do veículo. Assim, isolam-se as aceleraçôes laterais (Fig. 1-c) geradas por outros fatores não relacionados com as vibraçôes verticais do veículo relacionadas com as irregularidades nos pavimentos (manobras em curvas, frenagens, etc.).

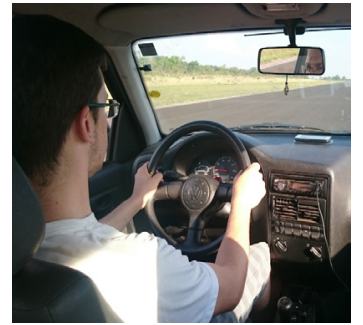

(a)

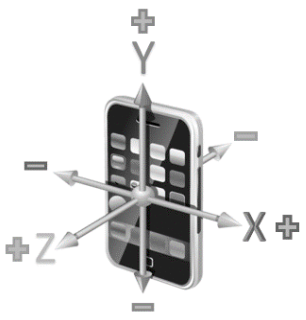

(b)

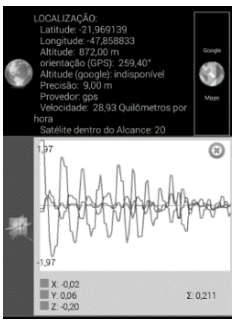

(c)

Figura 1 - (a) Smartphone fixado no painel de um veículo; (b) sistema de coordenadas de um smartphone;

(c) detalhe dos sinais de aceleração coletados por um aplicativo chamado AndroSensor: adaptada de [2].

Dada a importância do levantamento contínuo de defeitos, principalmente da irregularidade longitudinal de pavimentos, diversas pesquisas têm buscado preencher uma lacuna nos métodos existentes para essa avaliação: desenvolvimento de um sistema com o menor custo e maior praticidade e exatidão possíveis.

Alguns autores avaliaram o uso de acelerômetros e GPS (Global Positioning System) externos para a mensuração da irregularidade $[4,5,6]$. Recentemente, com vistas à redução de custos e possibilidade de coleta de dados a partir de processos colaborativos (crowdsourcing), passaram a ser utilizados dispositivos móveis para a avaliação da irregularidade longitudinal de pavimentos, em termos de IRI (International Roughness Index). Alguns buscam o cálculo direto do índice a partir dos sinais de aceleração verticais fornecidos por esses aparelhos $[4,7,8]$, enquanto outros analisam a correlação entre esse índice e os dados de aceleração $[9,10,11,12,13,14,15,16]$.

No cálculo do IRI, simula-se a passagem de um quarto de carro sobre um perfil de pavimento medido, conforme Fig. 2. O modelo matemático incorpora parâmetros de um veículo padrão, popu- 
larizado como Golden Car: pneu, mola e amortecedor (suspensão), massa não suspensa e massa suspensa. A simulação é realizada, por questôes práticas e técnicas, com a velocidade de $80 \mathrm{~km} / \mathrm{h}$ [17].

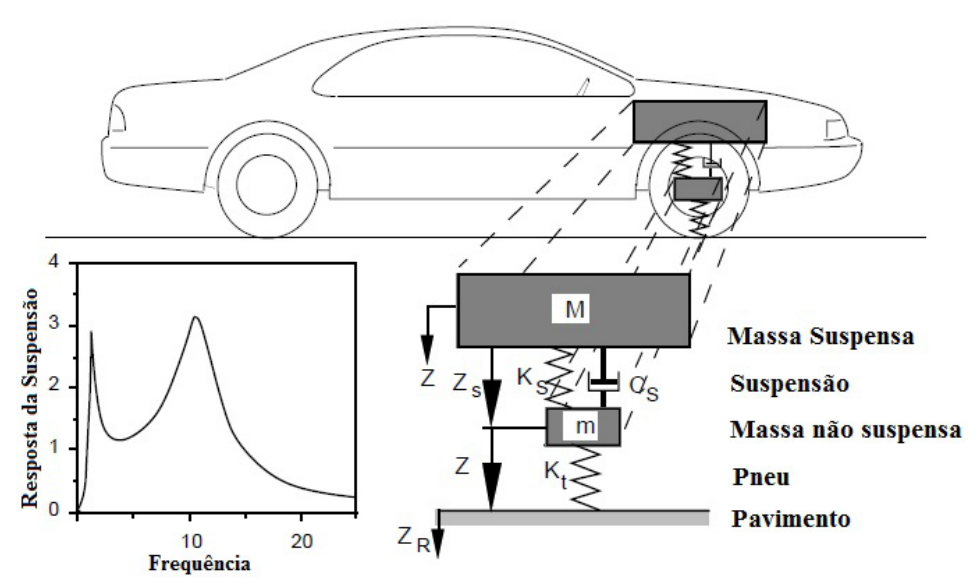

Figura 2 - Representação do modelo quarto de carro e gráfico de resposta da suspensão em diferentes frequências: adaptada de [18].

Para o cálculo direto do IRI a partir de dados fornecidos por dispositivos móveis, é preciso que os sinais de aceleração verticais coletados sejam tratados antes de serem duplamente integrados, em função do tempo, para a obtenção de deslocamentos verticais utilizados no cálculo do índice. $\mathrm{O}$ tratamento consiste na utilização de filtros digitais (isto é, sistemas que modificam os sinais do tempo discreto por meio de operaçóes matemáticas) para reduzir a influência de ruídos, caracterizados como sinais aleatórios produzidos pelos acelerômetros, que não estão relacionados com as vibrações do corpo do veículo, interferindo no sinal de saída.

Das pesquisas revisadas, não há detalhes sobre o processamento realizado para o cálculo do IRI a partir de dados de smartphones [10, 7, 8]. [8] citam a utilização de uma transformada de Fourier para a prevenção de erros de integração ocasionados por componentes de baixa frequência existentes em sinais fornecidos pelos smartphones. [7] comentaram apenas que o detalhamento do cálculo estava fora do escopo do artigo.

No processamento de sinais, é preciso considerar a escolha do filtro. Alguns podem causar atrasos consideráveis durante a filtragem dos sinais, devido à ordem do filtro, ou distorçóes decorrentes de respostas não lineares características do filtro utilizado. Esse efeito pode ser representado em termos de amostras ou em unidades de tempo [19]. Em geral, utilizam-se filtros passa-alta de janela curta [20].

Independente da técnica utilizada, ressalta-se que os dados fornecidos por dispositivos móveis não podem ser utilizados como as de um perfilômetro, mesmo que sejam obtidos valores de IRI próximos aos de perfilômetros. Isso porque o sistema é essencialmente do tipo resposta, ou seja, os sinais de saída são filtrados pelas características do veículo utilizado. Alguns estudos têm avaliado pavimen- 
tos com baixos níveis de irregularidade [7, 8]. Perfis mais irregulares, com menores comprimentos de onda (remendos, pequenos buracos e protuberâncias), podem denotar uma discrepância entre os índices obtidos por esses dois sistemas.

Por outro lado, é possível estimar a irregularidade a partir de correlaçôes com as medidas obtidas por equipamentos de referência. Nesse caso, [2] comenta que, assim como nos medidores do tipo resposta, a maior desvantagem do uso de smartphones seria a necessidade de um processo de calibração, já que os resultados variam de acordo com o modelo do veículo e sua velocidade, com o modelo de smartphone e aplicativo utilizados. Em sua pesquisa, [2] obteve valores de IRI a partir do método de Nível e Mira, de acordo com os procedimentos de calibração de medidores de irregularidade do tipo resposta da norma do [21]. A norma indica o uso de 20 trechos de calibração (bases) de 320 metros. Nesse caso, o autor estima que a calibraçáo demandaria em torno de um a dois meses para ser finalizada, de acordo com as condiçôes climáticas, disponibilidade de equipamentos e funcionários, dentre outros fatores.

Uma alternativa para o processo de calibração desse sistema seria a utilização dos aparelhos durante o levantamento de irregularidade realizado por outros sistemas, tidos como de referência, como os perfilômetros inerciais. Também é possível a aplicação desse mesmo procedimento quando perfilômetros inerciais passam por testes de verificação. Como explicam [17], tais equipamentos não passam por procedimentos de calibração como os medidores do tipo resposta, mas podem ter seus perfis comparados com outros métodos de referência (medidores estáticos).

Além dos problemas comuns aos medidores do tipo resposta, os dispositivos móveis apresentam algumas falhas que dificultam sua aplicação, principalmente se houver a intenção de utilizar os sinais de aceleração fornecidos para o cálculo do IRI. Uma delas é a limitação da taxa de aquisição de dados dos acelerômetros instalados nesses aparelhos. Uma baixa taxa de aquisição de dados pode causar a atenuação dos sinais de aceleração, tanto no domínio do tempo, quanto no domínio da frequência, sendo que o efeito de aliasing ocorrerá no momento em que a taxa de aquisição de dados for menor do que a taxa definida pelo teorema de Nyquist, segundo o qual um sinal analógico, limitado em banda, que foi amostrado, poderá ser reconstruído se a taxa de amostragem for maior ou igual a duas vezes a largura de banda do sinal original.

A atenuação das aceleraçóes resulta na subestimativa do nível de irregularidade dos pavimentos. Na presença do aliasing, os sinais de baixa frequência podem apresentar magnitudes maiores que as reais, quando observados no domínio da frequência, assim como baixas magnitudes em altas frequências. Deste modo, abordagens que buscam o cálculo direto do IRI, as quais necessariamente precisam realizar um tratamento dos dados no domínio da frequência, podem alcançar resultados que não correspondem com a realidade, tanto com valores superiores, quanto inferiores ao verdadeiro nível de irregularidade de um pavimento [22].

Segundo [23], diversos fatores podem influenciar na distribuição espectral dos dados relativos às vibraçôes de um veículo, como sua velocidade, as características de perfil do pavimento, as quais 
compreendem a quantidade e o comprimento de onda dos defeitos existentes no revestimento da estrada (remendos desnivelados, deformações permanentes, corrugações e panelas), bem como do próprio tipo de revestimento.

É preciso ter cautela com os resultados de IRI obtidos em recentes pesquisas. No estudo de [8], utilizaram-se três modelos de smartphones distintos. As taxas de aquisição de dados foram de $48 \mathrm{~Hz}$ para dois smartphones e $32 \mathrm{~Hz}$ para o terceiro. Como indicado por [2], que realizou diversos testes de vibração com três modelos de smartphones em laboratório e análises espectrais em três perfis de pavimentos, tais taxas sáo passíveis de erros nos cálculos de deslocamentos verticais e, consequentemente, no valor final do IRI. [2], no entanto, pondera que os resultados do cálculo do IRI dependem do método utilizado, do espectro em frequência dos pavimentos avaliados, da resposta dinâmica do veículo e do algoritmo de cálculo dos deslocamentos.

De acordo com [24], pesquisas futuras e projetos-piloto devem considerar métricas alternativas para a serventia de pavimentos. Argumenta que o IRI é o mais popular, por ser o índice internacional padrão. No entanto, comenta que não há nada mágico na relação entre IRI e serventia de pavimentos. Sugere o desenvolvimento de índices mais apropriados para esse sistema em busca de sua aplicabilidade.

[14] propuseram uma métrica de irregularidade baseada na aceleração, normalizada pela velocidade do veículo, a ser utilizada como base para a indicação preliminar de segmentos de pavimentos susceptíveis à degradação. A ideia é que, posteriormente, um perfilômetro possa ser enviado para a medição da irregularidade do pavimento com maior precisão. Nesse caso, haveria uma complementação entre levantamentos de controle (nível de rede) e levantamentos detalhados (nível de projeto).

Outra alternativa foi proposta por [25]. Em continuidade aos estudos realizados desde 2012, no Instituto de Pesquisas de Transportes da Universidade de Michigan (UMTRI - University of Michigan: Transportation Research Institute), observaram que os smartphones apresentam maior potencial quando utilizados para o prognóstico de categorias de classificação de irregularidade, ao invés de focar em dados exatos de IRI, devido à variabilidade dos sinais fornecidos, conforme os modelos de smartphones e veículos utilizados. Contudo, diferente da classificação de [10], que utilizou quatro categorias, sugerem a aplicação de três ou cinco categorias. Considera-se a última abordagem mais apropriada, pois possibilita a definição de uma categoria intermediária.

Alguns pesquisadores têm focado em estudos-piloto de coleta de dados colaborativa [26, 27]. Exemplo prático desse sistema é o StreetBump, aplicativo desenvolvido com auxílio público para o mapeamento de buracos e outros defeitos nas ruas de Boston, capital de Massachusetts. Sinais do acelerômetro e GPS fornecidos pelos smartphones dos usuários, que utilizam o aplicativo, são enviados a um servidor administrado pelo departamento de obras públicas de Boston.

Em princípio, existem duas abordagens para a coleta de dados colaborativa: 1) dados obtidos por frotas de veículos operados por agências de transpores ou 2) obtidos por veículos privados con- 
duzidos pelo público em geral. A razão para a distinção entre os veículos operados por agências e os privados é referente à participação do condutor. No último caso, seria necessária a iniciativa voluntária de condutores para a obtençáo dos dados. Uma das vantagens do uso de uma frota de veículos operados por agências é que, se o veículo é capaz de coletar informaçóes confráveis, isso exigiria somente um cuidado maior por parte do condutor, o que permitiria um controle maior da coleta de dados em relação àqueles obtidos pelo público em geral [24].

A participaçáo de usuários no levantamento de dados pode ser uma proposta promissora, devido ao potencial de coleta de um grande volume de informaçóes. Entretanto, além das dificuldades técnicas do sistema, seria preciso promover formas de incentivo aos usuários no uso de aplicativos desenvolvidos com esse fim, para que houvesse uma cobertura consistente do sistema. Outras dificuldades estariam relacionadas às montagens inadequadas e interação limitada com os condutores.

Outro problema na obtenção de dados em ambientes urbanos é a impossibilidade, salvo em vias específicas, da manutenção da velocidade do veículo, uma vez que os sinais de aceleração variam conforme essa variável, assim como ocorre em medidores do tipo resposta. As vibraçôes decorrentes de buracos ou outras irregularidades fornecem sinais de aceleração com uma variabilidade considerável, ou seja, a obtenção de padróes para identificação de defeitos não é trivial, pois demanda técnicas de filtragem. Nesse sentido, a aplicação de técnicas de processamento de sinais apresenta limitaçóes para a identificação de defeitos, como trincas, corrugaçóes, exsudação e desgastes, assim como a determinaçáo de grau de severidade dos defeitos ainda é um desafio, dada a variabilidade de diversos fatores, como o modelo do veículo, a pressão dos pneus, massa do veículo e a forma como é manobrado.

\section{Conclusóes}

Apesar da diversidade de pesquisas relacionadas ao monitoramento de pavimentos a partir de dispositivos móveis, com diferentes métodos, ainda é preciso vencer algumas barreiras para que o sistema ofereça confiabilidade para sua implantação, inclusive com a prescrição de normas e procedimentos padronizados. Por outro lado, também é notável a contribuição desses estudos, que já indicam a capacidade do uso dos aparelhos para a identificação de buracos em pavimentos urbanos com relativa acurácia, assim como uma alta correlaçáo com o nível de irregularidade de pavimentos em rodovias rurais.

Ressalta-se que o maior objetivo das pesquisas é a detecção de defeitos em pavimentos em tempo real, com redução de custos, maior praticidade e exatidão possível, o que não é uma tarefa simples. Ainda não há um método que, a partir dos sinais fornecidos por dispositivos móveis, possa individualmente solucionar o problema. Entretanto, apesar das dificuldades, com o avanço da eletrônica nesses dispositivos, que permitiu e instigou pesquisas correlatas, e da aplicação de técnicas de processamento, essa tecnologia apresenta potencial de aplicação nos próximos anos. 
É preciso ter claro o objetivo que se busca com tais medidas. Com um controle adequado, os dispositivos móveis já podem ser utilizados como indicativos da condição dos pavimentos em nível de rede, contribuindo para a alimentação de sistemas de gerência de pavimentos. Mesmo com limitações para apontar a diferença entre um buraco, um remendo desnivelado, deformações permanentes ou o nível de irregularidade em um pavimento, ainda assim, estará identificando um defeito que possivelmente deverá passar por algum tipo de manutenção, corretiva ou preventiva.

\section{Referências}

[1] FERNANDES JR., J. L.; ODA, S.; ZERBINI, L. F. Defeitos e Atividades de Manutençáo e Reabilitaçáo em Pavimentos Asfálticos. Apostila. Escola de Engenharia de São Carlos, USP. São Carlos, SP. 2011.

[2] BISCONSINI, D. R. Avaliaçáo da Irregularidade Longitudinal dos Pavimentos com Dados Coletados por Smartphones. Dissertação de Mestrado, Escola de Engenharia de São Carlos da Universidade de São Paulo. São Paulo, SP. 2016.

[3] TOMIYAMA, K.; KAWAMURA, A.; NAKAJIMA, S.; ISHIDA, T.; JOMOTO, M. A mobile profilometer for road surface monitoring by use of accelerometers. In 7 th Symposium on Pavement Surface Characteristics: SURF 2012, Norfolk. 2012.

[4] DOUANGPHACHANH, V.; ONEYMA, H. Estimation of road roughness condition from smartphones under realistic settings. In Proceedings of the 13th International Conference on ITS Telecommunications (ITST). 5-7. pp. 433-439. 2014.

[5] DU, Y.; LIU C.; WU D.; JIANG, S. Measurement of International Roughness Index by Using Z-Axis Accelerometers and GPS. Mathematical Problems in Engineering. v. I. Key Laboratory of Road and Traffic Engineering of the Ministry of Education, Tongji University, Shanghai 201804, China. 2014.

[6] NITSCHE, P; GEEM, C. V.; STUTZ, R.; MOCANU, I.; SJGREN, L. Monitoring ride quality on roads with existing sensors in passenger cars. In: Proceedings of the 26th ARRB Conference, Sydney, pp. 1-13. 2014.

[7] BUTTLAR, W. G.; ISLAM, S. Measurement of pavement roughness using an Android-based smartphone application. Transportation Research Board 93 ${ }^{\text {rd }}$ Annual Meeting, Washington D.C., U.S.A. 2014.

[8] HANSON, T.; CAMERON, C.; HILDEBRAND, E. Evaluation of low-cost consumer-level mobile phone technology for measuring international roughness index (IRI) values. Canadian Journal of Civil Engineering. pp. 819-827. doi:10.1139/cjce-2014-0183. 2014.

[9] HANSON, T. R.; CAMERON, C. Can a smartphone collect IRI data? Conference and Exhibition of the Transportation Association of Canada - Transportation: Innovations and Opportunities Fredection, NB, Canada. 2012.

[10] FORSLOF, L. Roadroid: Continuous road condition monitoring with smart phones. In IRF $17^{\text {th }}$ World Meeting and Exhibition, Riyadh, Saudi Arabia. 2013.

[11] ALESSANDRONI, G.; KLOPFENSTEIN L. C.; DELPRIORI S.; DROMEDARI M.; LUCHETTI G.; PAOLINI B. D.; SERAGHITI A.; LATTANZI E.; FRESCHI V.; CARINI A; BOGLIOLI A. Smartroadsense: Collaborative road surface condition monitoring. International Conference on Mobile Ubiquitous Computing, Systems, Services and Technologies, Ubicomm, pp. 210-215. 2014.

[12] JIMÉNEZ L. A.; MATOUT N. A low cost solution to assess road's roughness surface condition for Pavement Management. Revista Global de Negocios (RGN) del Institute for Business and Finance Research. TRB 2014 Annual Meeting. Proceedings of the 93th Annual meeting of the Transportation Research Board of the National Academies, n.3086, Washington, D.C., pp.1-16. 2014.

[13] ZENG, H.; SMITH B. L.; PARK. H.; PARKANY E. A Connected Vehicle-Based Application to Estimate Road Roughness. Poster presented in Connected Vehicle Infrastructure University Transportation Center - CVI UTC. Center for Transportation Studies - University of Virginia. Virginia, USA. 2014. 
[14] ZENG, H.; PARK, H.; FONTAINE, M.D.; SMITH, B.L.; MCGHEE, K.K. Identifying Deficient Pavement Sections Using an Improved Acceleration-Based Metric. Transportation Research Board of the National Academies, Washington, D.C. Aceito para publicação. 2015.

[15] JANANI, L.; SABU, P. Smartphone Based Pavement Condition Assesment. International Advanced Research Journal in Science, Engineering and Technology. v. 3. pp. 281-286. 2016.

[16] LAUBIS K., SIMKO V., SCHULLER A. Road Condition Measurement and Assessment: A Crowd Based Sensing Approach. Proceedings of the Thirty Seventh International Conference on Information Systems. Dublin, Ireland. pp. 11-14. 2016.

[17] SAYERS, M.W.; GILLESPIE, T. D.; PATERSON, W. D. O. Guidelines for Conducting and Calibrating Road Roughness Measurements. World Bank Technical Paper Number 46, 87 p. 1986.

[18] SAYERS, M. W.; KARAMIHAS, S. M. The little book of profiling. Ann Arbor: Transportation Research Institute, University of Michigan, USA. 1998.

[19] SLIFKA L. D. An accelerometer based approach to measuring displacement of a vehicle body. M.S. thesis, Dept. Elect. Comput. Eng., Michigan Univ., USA. 2004.

[20] DEL ROSARIO M. B.; REDMOND S. J.; LOVELL N. H. Tracking the Evolution of Smartphone Sensing for Monitoring Human Movement. Sensors, vol. 15, no. 8, pp. 18901 - 18933. 2015.

[21] DEPARTAMENTO NACIONAL DE ESTRADAS DE RODAGEM - DNER. Método do Nível e Mira para Calibraçáo de Sistemas Medidores de Irregularidade Tipo Resposta. DNER-ES 173/86. Ministério dos Transportes, Espírito Santo. 1986.

[22] BISCONSINI, D. R.; NUNEZ, J. Y. M.; NICOLETTI, R.; FERNANDES JR, J. L. Consideraçóes sobre o uso de smartphones para a avaliaçáo da irregularidade longitudinal de pavimentos. XXX ANPET, 2016, Rio de Janeiro. XXX ANPET. 2016.

[23] BISCONSINI, D. R.; NUNEZ, J. Y. M.; SANTOS, J. N. dos; FERNANDES JR., J. L. Análise Espectral dos Dados Coletados por Smartphones para a Avaliaçáo da Irregularidade Longitudinal dos Pavimentos. $45^{\circ} \mathrm{RAPv} /$ $19^{\circ}$ ENACOR / $1^{\circ}$ Fórum Rodoviário, de Trânsito e de Mobilidade, 2016, Brasília. Anais do 45 RAPv. 2016.

[24] DENNIS, E. P.; HONG, Q.; WALLACE, R; TANSIL, W.; SMITH M. Pavement Condition Monitoring with Crowdsourced Connected Vehicle Data. Transportation Research Record: Journal of the Transportation Research Board, n. 2460, Transportation Research Board of the National Academies, Washington, D. C., pp. 31-38. doi: 10.3141/2460-04. 2014

[25] BELZOWSKI B.; EKSTROM A. Evaluating Rodway Surface Rating Technologies. Technical Report. University of Michigan Transportation Research Institute and the Michigan Department of Transportation. 2015.

[26] BRIDGELALL, R.; RAHMAN, M. T.; TOLLIVER, D. D.; DALEIDEN J. F. Use of Connected Vehicles to Characterize Ride Quality. Transportation Research Board of the National Academies, Washington, D.C. 2016.

[27] GEEM, C. V.; BELLEN, M.; BOGAERTS, B.; BEUSEN, B.; BERLÉMONT, B.; DENYS, T.; MEULENAERE, P. D.; MERTENS, L.; HELLINCKX, P. Sensors on Vehicles (SENSOVO) Proof-of-concept for Road Surface Distress Detection with Wheel Accelerations and ToF Camera Data Collected by a Fleet of Ordinary Vehicles. $6^{\circ}$ Transport Research Arena 2016, Transportation Research Procedia. 2016. 